\title{
Duelo y recuperación tras el inicio de la psicosis.
}

Grief and recovery after the onset of psychosis.

\author{
Fátima Valencia Agudo ${ }^{\text {a }}$. \\ ${ }^{a}$ Psicóloga Interna Residente. Hospital Universitario Central de Asturias, Oviedo, España. \\ Correspondencia: Fátima Valencia Agudo (fatima-valencia@hotmail.es)
}

Recibido: 17/01/2013; aceptado: 06/08/2013

RESUMEN: El presente artículo trata acerca del
proceso de duelo que tiene lugar cuando una persona
sufre un episodio psicótico. Se plantean cuáles son
las pérdidas más frecuentes que tienen lugar y las re-
acciones del paciente ante ello. Además, se proponen
algunas ideas para un trabajo clínico centrado en la
elaboración de la pérdida y la reconstrucción de una
identidad más allá del síntoma.

PALABRAS CLAVE: Duelo, trastornos psicóticos, identidad, psicoterapia.
ABSTRACT: This article is about the grief process that takes place when a person suffers a psychotic breakdown. The most common losses and frequent patient reactions to them are described. Moreover, this article poses some ideas for a loss-centered psychotherapy that also works towards the identity reconstruction beyond the symptom.

KEY WORDS: Grief, psychotic disorders, identity, psychotherapy.

Agradecimientos:

A Javier Llamazares, Juan G. Haro, Carlos Veiga y Mónica Pérez por leer el texto y aportar sugerencias que me han resultado de gran utilidad.

\section{Introducción}

Para referirse al duelo, Neimeyer (1) hace referencia a una pérdida a lo largo del ciclo vital en diferentes ámbitos. Puede estar asociado a la muerte de una persona cercana (significado socialmente más reconocido), pero también a una separación conyugal o a la pérdida relacional de un familiar o de un amigo. También implica la pérdida de bienes materiales (por ejemplo en un robo o en un desastre natural) y la de capacidades mentales y físicas. Este autor reconoce similitudes en la forma en que las personas afrontan cada una de estas dificultades. Además, plantea que las pérdidas pueden ser evidentes, como es el caso de alguien que pierde un hijo, o más sutiles, como quien pierde la capacidad física con el paso de los años. Para Neimeyer, la falta de comprensión y la trivialización de la pérdida son factores que agravan el malestar y que se dan especialmente cuando esta no es socialmente tan saliente.

Cuando se trata de relacionar el duelo y la psicosis existen varias líneas de trabajo, si bien los estudios acerca del tema son escasos y se han realizado fundamentalmente en los últimos años. Por una parte, algunos artículos exploran cómo un proceso de duelo puede suponer un desencadenante para la aparición de un pri- 
ORIGINALES Y REVISIONES

mer episodio psicótico (2). Algunos de ellos hacen referencia concreta a la pérdida cultural que supone la inmigración (3). Por otro lado, otros autores se centran en el duelo al que da lugar el propio trastorno, es decir, aquello que la persona pierde (directa o indirectamente) como consecuencia de la psicosis (4-5). Este es el tema acerca del que trataremos en el artículo.

Wittmann y Keshavan (4) proponen que el duelo es algo habitual tras un diagnóstico de psicosis, una experiencia fundamental a la que el paciente ha de hacer frente. Asimismo, para Edwards y McGorry (6) una parte de la recuperación de la persona pasa por comprender y asimilar lo que le ha ocurrido.

Young, Bailey y Rycroft (5) plantean que en los servicios de salud mental no se suele trabajar directamente con el duelo en casos de trastorno mental grave. Añaden además que en muchos casos se psicopatologizan emociones comprensibles tales como la rabia, la tristeza o la confusión, tanto de la persona como de la familia. Los pacientes sienten un malestar importante al darse cuenta de cómo el trastorno afectará a sus vidas (7).

Este trabajo pretende dar cuenta de las pérdidas que sufre una persona tras un episodio psicótico, de las dificultades que tiene a la hora de recuperar lo que ha perdido así como de las posibles formas de afrontamiento.

\section{Características del duelo en la psicosis}

Para algunos autores el duelo en la psicosis es particularmente complicado y con frecuencia llega a ser patológico. Tari García y Ferrer Dufol (8) hablan de duelo acumulativo para referirse a las múltiples pérdidas en diversos ámbitos y en diferentes momentos de la evolución del trastorno. Además establecen tres tipos de factores que afectan a la elaboración del duelo: intrínsecos al tipo de duelo, factores microsociales y factores relacionados con la capacidad de los pacientes para afrontar la pérdida. Nos centraremos en el primer factor, ya que da cuenta de las características particulares que tiene el duelo en la psicosis.

En primer lugar, los autores plantean que el duelo en la psicosis es parcial, ya que la pérdida no es absoluta ni permanente. Esto da lugar a que la esperanza de recuperación dificulte paradójicamente la misma (9). En este sentido, este tipo de duelo se caracterizaría por la pérdida ambigua, concepto propuesto por Boss (10). Este hace referencia a un duelo que permanece sin resolver. Propone dos tipos de pérdidas ambiguas relacionales: cuando la persona está ausente física pero no psicológicamente (por ejemplo en una desaparición), o a la inversa, cuando el ser querido está presente de manera física pero ausente (o diferente) a nivel psicológico. Este último caso sería aplicable a la psicosis, tanto en lo que se refiere a la 
ORIGINALES Y REVISIONES

pérdida relacional que sufre la familia, como al propio paciente en el sentido de las capacidades o roles sociales que han cambiado. Añade que la pérdida ambigua implica ausencia de validación social y de ritualización.

Además, dada la evolución del trastorno por episodios, el duelo es recurrente, no termina nunca. Podría decirse que la persona y su entorno están siempre perdiendo.

Otro factor a considerar es la multiplicidad de las pérdidas, aspecto al que se hará referencia en el siguiente apartado.

Por otra parte, el duelo es compartido, es decir, la familia también añora lo que ha quedado atrás e intenta adaptarse a la nueva situación. Las personas cercanas al paciente han de aceptar que su hijo, pareja o padre, quizás no vuelva a ser como antes y por ello hay que tenerles muy en cuenta. Los procesos de duelo de la persona y de la familia están interrelacionados, de forma que el entendimiento que cada parte tiene del trastorno y las expectativas acerca de la recuperación influyen en la otra parte y se adaptan entre sí (11).

Otra cuestión que proponen Tari y Ferrer es el segundo duelo, entendido como los esfuerzos que el paciente ha de hacer por normalizar su vida, tras haber estado durante algún tiempo en el rol de enfermo. Es algo importante a tener en cuenta, ya que en este momento puede perder la atención y el cuidado del entorno o tener que enfrentarse a determinadas situaciones y tareas para las que no se siente preparado.

Estos autores no son los únicos en hablar de las complicaciones del duelo en la psicosis. Doka (12) propuso el término disenfranchised grief para referirse a una pérdida que no es socialmente validada ya que no se percibe como tal por parte de la sociedad.

Por último, y retomando los planteamientos de Neimeyer (1), el duelo en la psicosis puede complicarse por la falta de comprensión del entorno, ya sea hacia el paciente o hacia la familia. Al igual que ocurre en ciertas pérdidas, como la de la pareja o el trabajo, no existen rituales en la comunidad que ayuden a la persona a elaborar el duelo y legitimizar su dolor.

Por todos los factores mencionados, este tipo de duelo es complejo y ha de abordarse con cautela haciéndose muchas preguntas. ¿Cuándo está claro que el paciente ha de hacer un duelo por su actividad laboral? ¿Cómo influye en la evolución el que la persona y su familia tengan altas expectativas de recuperación? ¿Y cómo lo hace el que acepten sin más la pérdida de una vida normalizada? ¿Es positivo que un paciente con importantes dificultades cognitivas o sociales se esfuerce en mejorar estas cuestiones? ¿O solo le llevará a la frustración y es mejor que se adapte a sus limitaciones? Preguntas con difícil respuesta y en todo caso diferentes según la persona. 
ORIGINALES Y REVISIONES

\section{Pérdidas en la psicosis}

¿Qué pérdidas se producen tras un episodio psicótico, especialmente tras el primero? En general, estas se dan en diferentes ámbitos, unas son concretas y otras de tipo simbólico. Como se mencionó en la introducción, el duelo puede estar relacionado con la pérdida de un ser querido, del trabajo, de la pareja, de ciertas capacidades, etc. En el caso de la psicosis puede que estemos hablando de todo ello a la vez.

Algunas estadísticas dicen que un tercio (33\%) de los pacientes que han sufrido un primer episodio psicótico pierden su empleo o abandonan su actividad formativa (13). Ochoa et al. (14) publicaron recientemente un estudio acerca de la autopercepción del estigma en personas diagnosticadas de psicosis. Una de las áreas que más preocupaban a los pacientes era la de pérdida de roles sociales. En este sentido los participantes hicieron referencia a la pérdida de relaciones (amistades, pareja, posibilidad de tener hijos) y de actividades ocupacionales (empleo, formación). Algunos comentarios que los autores reflejan en el artículo (pág. 484) se relacionan no sólo con haber perdido algo, sino también con la desesperanza de poder recuperarlo. El estudio cualitativo de Palma et al. (15) muestra resultados similares. Seguramente no se trata solo de un trabajo concreto o de una pareja o de un grupo determinado de amigos, sino de la imposibilidad de conseguir cualquier trabajo, de tener una pareja y amistades. Puede que la clave sea el sentimiento de pérdida de la capacidad y del reconocimiento social de la misma.

En esta línea, Lewis (16) propone que uno de los factores de riesgo de suicidio en la esquizofrenia es haber tenido un mejor funcionamiento premórbido y mayor nivel de inteligencia. Pone estos aspectos en relación con mayores expectativas vitales que pasan a considerarse como inalcanzables. Sin embargo, esto no debería ser considerado por los profesionales de Salud Mental como un hándicap, ni supone que pacientes con peor funcionamiento premórbido tengan un pronóstico más favorable. Sí que implica que determinadas personas pueden encontrar mayores dificultades a la hora de elaborar el duelo y seguramente requieren ayuda psicológica específica en este sentido.

Como se planteaba en el anterior apartado, el duelo en la psicosis es múltiple (8). Se proponen cuatro tipos de pérdida (pág. 135):

- Pérdida de capacidades y de determinados aspectos que definían a la persona (valores, aficiones, etc.).

- Pérdidas materiales, incluyendo en este apartado el trabajo, posesiones, el estatus socioeconómico o incluso el propio domicilio.

- Pérdidas relacionales, ya sea de familiares, pareja o amistades.

- Pérdida de proyectos vitales o experiencias asociadas a diferentes momentos del ciclo vital normal; se trata de aquello que el paciente no llegará a vivir a causa del problema que sufre. 
En relación a este último punto, también Young, Bailey y Rycroft (5) hablan de la pérdida de las expectativas de futuro: logros personales, completar los estudios, conseguir la independencia, tener hijos, etc. Destacan además el cambio que se produce en la relación de pareja, ya que de un status igualitario se pasa a una relación complementaria en la que uno es el cuidador y otro el enfermo. Esta situación puede implicar un proceso de duelo para ambos.

Otra pérdida importante es la que puede tener lugar en el ámbito de la familia. Por un lado, el individuo pierde el nivel de independencia que había conseguido respecto al núcleo familiar, tanto en lo económico como en otros aspectos. Por otra parte, y como muestra la práctica clínica, es habitual que el paciente reciba críticas por parte de su entorno, que en ciertos casos no llega a comprender la situación por la que pasa la persona. La otra cara de la moneda es la familia, que tiene que organizarse para hacer frente a una situación que les afecta a nivel social, emocional y económico. Atkinson (17) estudió las reacciones de padres cuyos hijos habían sido diagnosticados de esquizofrenia. Los resultados pusieron de manifiesto que se encontraban en una situación de duelo permanente.

Se han mencionado muchos tipos de pérdidas que tienen un impacto doloroso en la vida de una persona. Pero quizás la pérdida más importante y que subyace a todas las demás en un caso de psicosis, es el duelo que el paciente ha de hacer por su identidad. De repente cambia el sentido de sí mismo que se va construyendo en función de las interacciones con el entorno y de las actividades habituales. En relación con esto, una familia comenta en consulta que su hijo les dijo "ya no tengo trabajo ni tengo pareja, no soy nadie". Una chica que sufre un primer brote psicótico pregunta “¿volveré a ser la de antes?”. Wittman y Keshavan (4) relatan un caso en el que destacan la pérdida del Self previo; al hablar de las pérdidas que ha tenido, la paciente dice que la mayor de todas ellas es la de su identidad personal.

Además, es fundamental tener en cuenta las alteraciones en la identidad que forman parte del propio trastorno. Entre ellas están la disminución del sentido de sí mismo y del sentido de agencia, así como la alteración de la autoconciencia corporal natural (18).

De esta forma, podemos hacernos una idea del grado de ruptura con el self previo y de la dificultad para reconstruir una nueva identidad que integre la anterior así como la situación y la experiencia actual.

\section{Formas de expresión del duelo}

Es difícil saber el porcentaje de pacientes que sufre un duelo complicado ante la psicosis. Por una parte, muchos de ellos tienen dificultades para la comunicación 
y por otra parte no se les suele preguntar explícitamente. En algunos casos es más evidente, ya que el individuo habla de la pena por lo que ha perdido o de la desesperanza que siente al pensar que nunca alcanzará sus objetivos. En otras ocasiones solo podemos inferir que lo que hace o dice una persona tiene que ver con un proceso de duelo (además de con otros factores relacionados con el trastorno).

Addington et al. (19) establecen que la depresión y el suicidio son las respuestas más frecuentes tras el primer episodio psicótico. Lewis (16) recoge una serie de estadísticas acerca de estas cuestiones en pacientes diagnosticados de esquizofrenia. Según algunas investigaciones, entre el 60 y el $80 \%$ presentan uno o más episodios depresivos y el 55\% han tenido intentos de suicidio (20-21). La tasa de suicidio es entre 20 y 50 veces mayor que en la población general (22). En relación a estos datos, Lewis destaca que en los últimos años ha aumentado la tasa de suicidio respecto al inicio del siglo pasado, lo que asocia con el aumento del tratamiento psicofarmacológico en detrimento de la relación terapéutica.

Birchwood, Iqbal y Upthegrove (23) ponen en relación las pérdidas sociales con estos síntomas depresivos, y lo hacen a través del constructo "depresión post psicótica". Hace referencia a la posible reacción de un individuo ante la psicosis, entendida esta como un evento vital. En una investigación (24), los autores determinaron que el $36 \%$ de los pacientes de su muestra habían desarrollado depresión post psicótica. Estas personas eran conscientes de las pérdidas que habían tenido y se mostraban más pesimistas respecto al futuro que aquellos que no estaban deprimidos. En este sentido, Kim, Jayathilake y Meltzer (25) encontraron que uno de los factores de riesgo de suicidio con más peso es la desesperanza.

En personas con este tipo de síntomas resulta relativamente fácil identificar el duelo, pero hay otros casos en los que no es tan evidente. Lafond (26) plantea que el duelo puede expresarse a través de la ira, la frustración, el letargo o como más síntomas del trastorno mental.

Algunos pacientes pueden mostrarse irritables o incluso agresivos cuando ven que tienen dificultades para realizar determinadas actividades que antes podían hacer. Un ejemplo podría ser el de un paciente ingresado en una Comunidad Terapéutica que se orina con frecuencia en la cama y los días que esto sucede se muestra más irascible y vociferante. Cabría plantearse si esta reacción tiene que ver con la pérdida del control de esfínteres y el sentimiento de incapacidad que esto puede conllevar.

Otra opción es que el individuo desarrolle un delirio compensatorio de forma que, en palabras de Linares (27), una nueva identidad basada en lo sintomático sustituya a la maltrecha identidad previa. Este no es el único autor que realiza este planteamiento. Puede verse cierto paralelismo con el paso de la fase de trema a la fase apofánica de Conrad (28); en esta última aparece la conciencia de significación anormal, que de alguna forma explica las experiencias inespecíficas de 
ORIGINALES Y REVISIONES

cambio y desintegración de la fase anterior. Lewis (16) también propone que los síntomas positivos a veces son una estrategia para encontrar sentido y reconstruir la identidad. Tari García y Ferrer Dufol (8) hablan de funcionamiento psicótico omnipotente o duelo por el camino equivocado para referirse al uso de síntomas psicóticos como forma de afrontamiento de situaciones difíciles y dolorosas. Este puede que sea el caso de J., un paciente de 50 años con multitud de ingresos hospitalarios y diagnóstico de esquizofrenia paranoide. En la historia puede verse que el motivo de ingreso en las primeras ocasiones fueron conductas antisociales e ideación delirante poco estructurada. Con el paso de los años, y en un contexto de escaso apoyo familiar y social y con nulas fuentes de autosatisfacción, J. desarrolla una temática delirante en la que se representa como una especie de héroe de guerra que ha viajado y luchado en múltiples países. En el momento de la valoración, se siente perseguido por una red de ladrones cuya trama está a punto de destapar. Además, en esta identidad sintomática, el paciente se casó y tuvo dos hijos. El delirio incluye prácticamente todo lo que no existe en la vida real.

\section{El contexto como modulador del duelo}

Si bien son evidentes las dificultades que una persona con diagnóstico de psicosis puede tener para llevar una vida normalizada, es fundamental tener en cuenta cómo el contexto clínico-social en el que es atendida y en el que vive determina en gran parte el grado de recuperación que es posible alcanzar. Y esto por supuesto va a influir en el proceso de duelo.

González Pardo y Pérez Álvarez (29) mencionan dos estudios transculturales patrocinados por la OMS que pusieron de manifiesto una mejor evolución y una menor interferencia en el funcionamiento de la psicosis en los países en vías de desarrollo. Para los autores, este fenómeno se produce por la mayor normalización del episodio y por el mantenimiento del rol social de la persona. En nuestro contexto, por el contrario, se dan las condiciones para que el episodio se cronifique: tratamiento psicofarmacológico (generalmente de por vida), hospitalización, abandono de la actividad laboral o formativa, rechazo o sobreprotección por parte del entorno, etc.

Otro factor que influye en la evolución del paciente y en el grado en que pueda mejorar su funcionamiento es la integración laboral. García et al. (13) destacan una serie de ventajas de la actividad laboral, entre las cuales está el ampliar la red social, mejorar las relaciones familiares, facilitar la independencia y proporcionar un sentido de identidad. Lastra et al. (30) muestran que aquellos pacientes que participaron en un programa de inserción laboral tuvieron un importante descenso 
en el número de días de hospitalización por año. A pesar de ello, los programas de formación y empleo son cada vez más escasos. Lo mismo puede decirse de los pisos tutelados, que suponen un paso previo a la vida independiente. Por poner un ejemplo, en la ciudad de Oviedo, perteneciente al área sanitaria IV de Asturias y que engloba a una población de 342.336 habitantes (31), existen únicamente dos pisos tutelados, con tres plazas para mujeres y cuatro para hombres.

Cabe también tener en cuenta los efectos secundarios de los tratamientos antipsicóticos. Estos se producen a nivel cognitivo (32), físico $(30,33)$, emocional y motivacional (30) y afectan a las relaciones interpersonales, a la capacidad de trabajar y formarse, a la percepción de si mismo y contribuyen al estigma social (33).

En definitiva, el contexto en el que nos encontramos no parece el más adecuado para que la persona que sufre un episodio psicótico recupere una vida normalizada. Esto tiene una influencia evidente a nivel emocional, ya que las pérdidas son mucho mayores y las expectativas de futuro se reducen.

\section{Elaboración del duelo y reconstrucción de la identidad}

Como ya se mencionó previamente, el duelo tras un episodio psicótico no es algo en lo que los profesionales de salud mental suelan centrarse específicamente. Cada vez se destaca más la importancia de hacerlo, en primer lugar para aliviar el sufrimiento de la persona y en segundo lugar porque el duelo puede llevar a estados de depresión postpsicótica (23), al abandono de los servicios (34) o aumentar el riesgo de suicidio (16).

Wittman y Keshavan (4) aplican las tareas del duelo propuestas por Worden (35) al duelo por el trastorno mental. En este caso, las tareas podrían ser aceptar y llorar la pérdida del self previo, adaptarse a un entorno que ha cambiado y por último llevar a cabo una reconstrucción de la identidad que recoja la situación anterior, los cambios que se han producido y también fortalezas y dificultades.

Andresen, Oades y Caputi (36) proponen cuatro componentes del proceso de recuperación, basándose en un estudio empírico de la experiencia de los pacientes:

- Mantener la esperanza.

- Re-establecer una identidad positiva.

- Construir una vida con sentido.

- Asumir responsabilidad y control.

Shepherd, Boardman y Slade (37) recogen estos aspectos como parte fundamental de su modelo de recuperación. No hacen referencia a una "recuperación clínica", sino a la recuperación de la vida o "recuperación social".

Para muchos autores, el primer paso es la comprensión y la validación de las emociones por parte de los profesionales $(5,38)$, y por supuesto del entorno. En la 
ORIGINALES Y REVISIONES

práctica habitual nos encontramos con pacientes y familias con graves problemas, tanto a nivel clínico como psicosocial. Esto hace que la resolución de ciertos aspectos centre nuestra atención, dejando de lado su experiencia subjetiva, sus fortalezas y sus deseos y preferencias. Young, Bailey y Rycroft (5) plantean hablar abiertamente del dilema que surge entre llorar la pérdida o seguir con la vida, así como normalizar tanto los momentos de dolor como los de sensación de competencia.

Otro proceso importante es el de dar sentido a lo que ha pasado. Un episodio psicótico es algo que habitualmente rompe con la historia personal anterior, por lo que es importante reconstruir lo ocurrido aportando un significado y tratando de dar una continuidad a la experiencia. Wittman y Keshavan (4) piensan que la explicación de lo sucedido ha de integrarse en la visión que se tiene de uno mismo y del mundo. Lewis (16) propone el concepto de usable insight (podría traducirse como insight útil), que consiste en la toma de conciencia de las dificultades pero también de las fortalezas y de los objetivos que uno puede alcanzar. El pensar que uno es un "enfermo mental" en manos de los profesionales de salud mental seguramente no puede considerarse un insight útil, como tampoco lo es el sentirse perfectamente capacitado y atribuir todos los males a un ente externo. Para esta autora, el propio proceso de insight implica un duelo, que considera una experiencia necesaria para evitar el sufrimiento que genera proponerse metas que difícilmente serán conseguidas.

El planteamiento de Lisa Lewis acerca del insight tiene que ver con el concepto de conciencia de enfermedad. Así, citando a Amador, Friedman y Kasapis (39), define insight como la conciencia de la enfermedad y de sus síntomas, de las consecuencias sociales de la misma y de la necesidad de tratamiento. Otros autores coinciden en la importancia de dar sentido a la experiencia y ser consciente de las limitaciones, pero sin imponer un modelo médico explicativo. White y Epston (40), desde la perspectiva de las terapias narrativas, destacan la importancia de pasar de "definiciones expertas" a "definiciones populares". Las primeras ignoran el contexto del problema y la influencia (ya sea positiva o negativa) de las personas sobre el mismo. Más recientemente, Larsen (41) plantea que en el ámbito de la salud mental cada persona (y cada clínico) elabora su propia reconstrucción de lo ocurrido. Enfatiza el sentido de agencia de cada paciente a la hora de llevar a cabo este proceso y propone el término bricolage para referirse a cómo alguien elabora su propia teoría a partir de las diferentes explicaciones culturales disponibles. De esta forma, critica el término "narrativa de enfermedad" y desarrolla en su lugar el de "sistema de explicación". Romme y Escher (42) realizan una propuesta similar en su libro Dando sentido a las voces, en el que se centran en las alucinaciones auditivas. En definitiva, lo verdaderamente relevante es que la explicación que el paciente se da tenga consecuencias adaptativas, coincida o no con lo que psicólogos clínicos y psiquiatras piensen.

Finalmente, es fundamental llevar a cabo un proceso de reconstrucción de la identidad. Parte de este importante trabajo seguramente se produzca cuando la per- 
ORIGINALES Y REVISIONES

sona encuentre un significado a la experiencia por la que ha pasado. Pero además, ha de establecer nuevas metas de dificultad progresiva que orienten su vida y proporcionen un sentido a su existencia. De esta forma, la nueva identidad se basará en más aspectos que aquellos relacionados con el trastorno o con los problemas relacionales. Uno de los autores que destaca los factores positivos del paciente y de la familia es Marley (43), que trabaja desde la perspectiva de la narrativa y las fortalezas. Su planteamiento contrasta con los modelos de déficit como son la psicoeducación y las intervenciones basadas en la emoción expresada.

Una de las metas que más valor puede tener en la recuperación es la relacionada con el empleo (37). Para Sheperd y sus colaboradores, trabajar supone la salida del rol pasivo de enfermedad y proporciona un sentido positivo a la identidad. Otro paso importante consiste en reestablecer relaciones con otras personas (5), ya que la identidad está asociada a las interacciones sociales. Como ya se mencionó, es habitual que el episodio psicótico tenga consecuencias negativas sobre las relaciones. Shepherd, Boardman y Slade (37) plantean que las personas que rodean al paciente también han de hacer su propio duelo. La familia ha de (re)encontrar nuevas formas de interacción que no se basen únicamente en las dificultades.

\section{Conclusiones}

Parece fundamental prestar atención a los procesos de duelo personales y familiares cuando un individuo sufre un episodio psicótico. Quizás el momento más importante sea el inicial, cuando los problemas emocionales, familiares, cognitivos y sociales no se han cronificado.

Sin embargo, el cambio de foco atencional no debe ser solo del clínico que atiende al paciente, sino que para que se produzca la verdadera recuperación debe haber "un cambio en la cultura, además de en la organización de los servicios" (37, pág. 19).

BIBLIOGRAFÍA:

(1) Neimeyer R. Aprender de la pérdida. Barcelona: Paidós, 2007.

(2) Gamo Medina E, Pazos Pezzi P. Reflexiones sobre duelo precoz y psicosis. Presentado en Congreso de la Asociación Castellano-Manchega de Salud Mental (AEN). Talavera de la Reina (Toledo), 2006.

(3) Martínez Moneo M y Martínez Larrea A. Patología psiquiátrica en el inmigrante. An Sist Sanit Navar 2006; 29 (Supl. 1): 63-75. 
(4) Wittmann D, Keshavan M. Grief and Mourning in Schizophrenia. Psychiatry 2007; Vol. 70, No. 2, pp. 154-166.

(5) Young J, Bailey G, Rycroft P. Family grief and mental health: a systemic, contextual and compassionate analysis. ANZJFT 2004; 25(4), 188-197.

(6) Edwards J, McGorry P. La intervención precoz en la Psicosis: Guía para la creación de servicios de intervención. Madrid: Fundación para la Investigación y el Tratamiento de la Esquizofrenia y otras psicosis, 2004.

(7) McGlashan TH. Recovery style from mental illness and long-term outcome. J Nerv Ment Dis 1987; 175, 11, 681-685.

(8) Tari García A, Ferrer Dufol C. Duelo y recuperación. En: Hernández Monsalve M y Nieto Degregori MP, coords. Psicoterapia y rehabilitación de pacientes con psicosis. Madrid: Grupo 5, 2011, págs. 127-150.

(9) Boss P. La pérdida ambigua. Barcelona: Gedisa, 2001.

(10) Boss P. Ambiguous Loss. In: Walsh, F, McGoldrick, M, editors. Living Beyond Loss. NY: Norton, 1991.

(11) Patterson P, Birchwood M, Cochrane R. Preventing the entrenchment of high expressed emotion in first episode psychosis: early developmental attachment pathways. Aust N Z J Psychiatry 2000, 34 (suppl.), S191.

(12) Doka KJ. Disenfranchised Grief: Recognizing Hidden Sorrow. MA: Lexington Books, 1989.

(13) García J, Peñuelas E, de las Heras B, Menéndez I, Díez ME. La formación y el empleo. Instrumentos básicos para la integración de las personas con trastornos psicóticos. Rev. Asoc. Esp. Neuropsiq. 2000; vol. XX, n. ${ }^{\circ}$ 73, pp. 9-22.

(14) Ochoa S, Martínez F, Ribas M, García-Franco M, López E et al. Estudio cualitativo sobre la autopercepción del estigma social en personas con esquizofrenia. Rev. Asoc. Esp. Neuropsiq. 2011; 31 (111), 477-489.

(15) Palma C, Ferrer M, Farriols Ny Cebrià J.La recuperación en la psicosis temprana: los procesos de duelo y el sentido de la experiencia psicótica. Anales de Psiquiatría 2006; Vol. 22, №6, pp. 296-305.

(16) Lewis L. Mourning, insight and reduction of suicide risk in schizophrenia. Bull Menninger Clin 2004; 68(3): 231-44.

(17) Atkinson SD. Grieving and Loss in Parents with a Schizophrenic Child. Am J Psychiatry 1994; 151, 8: 1137-1139.

(18) Pérez Álvarez M, García Montes JM y Sass L. La hora de la fenomenología en la esquizofrenia. Clínica y Salud 2010; Vol. 21, n³. Págs. 221-233.

(19) Addington J, Williams J, Young J, Addington D. Suicidal behaviour in early psychosis. Acta Psychiatr. Scand. 2004; 109, 116-120.

(20) Fenton WS. Depression, suicide, and suicide prevention in schizophrenia. Suicide Life Threat Behav 2000; 30, 34-49.

(21) Wagstaff AJ, Perry CM. Clozapine in prevention of suicide in patients with schizophrenia or schizoaffective disorder. CNS Drugs 2003; 17, 273-280.

(22) Pinikahana J, Happel B, Keks NA. Suicide and schizophrenia: A review of the literature for the decade (1990-1999) and implications for mental health nursing. Issues Ment Health Nurs 2003; 24, 27-44.

(23) Birchwood M, Iqbal Z, Upthegrove R. Psychological pathways to depression in schizophrenia. Studies in acute psychosis, post psychotic depression and auditory hallucinations. Eur Arch Psychiatry Clin Neurosci 2005; 255, 202-12. 
(24) Birchwood M, Iqbal Z, Chadwick P, Trower P. Cognitive approach to depression and suicidal thinking in psychosis. Br J Psychiatry 2000; 177, 516-521.

(25) Kim CH, Jayathilake K, Meltzer HM. Hopelessness, neurocognitive function, and insight in schizophrenia: Relationship to suicidal behavior. Schizophr Res 2003; 60, 71-80.

(26) Lafond V. Oration on the occasion of the 21st Anniversary of AREFEMI, in Melbourne, 2000.

(27) Linares JL. Identidad y narrativa. Madrid: Paidós Terapia Familiar, 1996.

(28) Conrad K. La esquizofrenia incipiente. Madrid: Fundación Archivos de Neurobiología, 1997.

(29) González Pardo H, Pérez Álvarez M. La invención de trastornos mentales. Madrid: Alianza Editorial, 2007.

(30) Lastra I, Arbildua C, Polo MA, Ruiz A, Verdugo S. Reducción de la hospitalización psiquiátrica en personas participantes en programas de inserción laboral. Informaciones Psiquiátricas Tercer trimestre 2009. Número 197.

(31) Servicio de Salud del Principado de Asturias. Memoria anual, 2011 (en línea). http:// www.asturias.es/Astursalud/Ficheros/AS_SESPA/As_Organizacion/MEMORIA\%20SESPA\%202011/ memoria\%20Sespa\%202011\%20multimedia.pdf.

(32) Valverde Eizaguirre MA. Una crítica a la teoría del déficit cognitivo en la esquizofrenia. Rev. Asoc. Esp. Neuropsiq. 2012; 32 (115), 521-545.

(33) Ross C, Read J. Los fármacos antipsicóticos: mitos y realidades. En Read J, Mosher L, Bentall R, coords. Modelos de locura. Barcelona: Herder, 2006.

(34) Tait L, Birchwood M, Trower P.Predicting Engagement with Services for Psychosis: Insight, symptoms and recovery style. Br J Psychiatry 2003; 182, 123-128.

(35) Worden JW. El tratamiento del duelo. Asesoramiento psicológico y terapia. Barcelona: Paidós, 1998.

(36) Andresen R, Oades L, Caputi P. The experience of recovery from schizophrenia: towards an empirically validated stage model. Austr N Z J Psychiatry 2003; 37, 586-594.

(37) Shepherd G, Boardman J, Slade M. Hacer de la recuperación una realidad. Revisión de la edición en castellano: Bono del Trigo A, Fernández Regidor G, García-Cubillana de la Cruz P, Mena Jiménez A, Trillo Gallo E, Vega Monclús E. Servicio Andaluz de Salud. Consejería de Salud. Junta de Andalucía, 2008. Disponible en http://www.centreformentalhealth.org.uk/pdfs/Hacer_Recuperacion_ Realidad.pdf.

(38) Miller, FM. Grief Therapy for Relatives of Persons With Serious Mental Illness. Psychiatric Services 1996; 47, 6: 633-637.

(39) Amador XF, Friedman JH, Kasapis C. Suicidal behavior in schizophrenia and its relationship to awareness of illness. Am J Psychiatry 1996; 153, 1185-1188.

(40) White M, Epston D. Medios narrativos para fines terapéuticos. Paidós. Barcelona, 1993.

(41) Larsen JA. Finding meaning in first episode psychosis: Experience, agency and the cultural repertoire. Med Anthropol Q 2004; 18(4), 447-471.

(42) Romme M, Escher S. Dando sentido a las voces. Guía para los profesionales de la salud mental que trabajan con personas que escuchan voces. Madrid: Fundación para la investigación y el tratamiento de la esquizofrenia y otras psicosis, 2005.

(43) Marley J. Family therapy and schizophrenia: A developing model for practice. J Fam Psychother 1999; 10, 1-14. 